\title{
In reply: Analysis of perioperative antibiotic administration in electronic medical records: correlations among patients addressed by analyzing control chart data using the batch means method
}

\author{
Alexander M. Hincker, MD • Arbi Ben Abdallah, DES, PhD • Michael Avidan, MBBCH • \\ Penka Candelario, BS • Daniel Helsten, MD
}

Received: 30 August 2017/Accepted: 8 September 2017/Published online: 15 September 2017

(C) Canadian Anesthesiologists' Society 2017

\section{To the Editor,}

We appreciate Dr. Dexter's interest in our study, "Electronic medical record interventions and recurrent perioperative antibiotic administration: a before-and-after study," ${ }^{1}$ as expressed in his recent letter. ${ }^{2}$ We agree with his assessment that our analysis oversimplified the situation by treating each case as an independent random sample and not accounting for correlations between patients, providers, and procedures. We also agree that attempting to rule out, or account for, such correlations is not feasible in this study. As such, per his recommendation, we have repeated our analysis to show a side-by-side comparison of the results using our initial approach compared with the summary measure method, using each month of cases as the unit of measure.

The results of this new analysis are shown in the Table. The top row treats each individual dose of antibiotic as a sample, as in our initial analysis. We then compared the differences in the proportions of correctly administered doses before the interventions (12 months, $n=1,367$ cases) and after the interventions ( 8 months, $n=1,004$ cases) using Chi-square analysis. The bottom row of the table treats each month as the unit of measure. We then compared the difference in the proportions of correctly administered doses per month before the intervention ( $n=12$ months) and after the intervention

Table Analysis of results using individual cases $v s$ months of cases as the unit of analysis

\begin{tabular}{lllll}
\hline Dose & First Doses & Second Doses & Third Doses & All Repeated Doses \\
\hline Difference in proportions of & $2.7 \%$ & $21.1 \%$ & $25.5 \%$ & $20.6 \%$ \\
compliant doses (individual) & $(95 \% \mathrm{CI}, 0.01 \%$ to & $(95 \% \mathrm{CI}, 17.3$ to $24.9 \%$, & $(95 \% \mathrm{CI}, 15.0 \%$ to & $(95 \% \mathrm{CI}, 17.0 \%$ to \\
& $4.8 \%, P<0.001)$ & $P<0.001)$ & $36.0 \%, P<0.001)$ & $24.2 \%, P<0.001)$ \\
Difference in proportions of & $1.9 \%$ & $20.5 \%$ & $28.0 \%$ & $20.6 \%$ \\
compliant doses (monthly) & $(95 \% \mathrm{CI},-0.9 \%$ to & $(95 \% \mathrm{CI}, 16.2 \%$ to & $(95 \% \mathrm{CI}, 13.3 \%$ to & $(95 \% \mathrm{CI}, 16.5 \%$ to \\
& $4.7 \%, P=0.20)$ & $24.7 \%, P<0.001)$ & $42.7 \%, P=0.002)$ & $24.8 \%, P<0.001)$
\end{tabular}

Comparisons are made between all cases before and after implementation of the electronic medical record interventions. Monthly comparisons include 12 months before the interventions $(n=12$ months) and eight months after the interventions $(n=8$ months). Individual level comparisons include 12 months before the interventions $(n=1,367$ first and second doses, 184 third doses, 1,551 total repeated doses) and 8 months after the interventions ( $n=1,004$ first and second doses, 132 third doses, 1,136 total repeated doses). $P$ values are for the Chi-square test for individual level proportions and for the two-tailed, unpaired $t$ test for pooled monthly data

$\mathrm{CI}=$ confidence intervals

A. M. Hincker, MD (ه) - A. Ben Abdallah, DES, PhD .

M. Avidan, MBBCH - P. Candelario, BS - D. Helsten, MD

Department of Anesthesiology, Washington University in Saint

Louis, Saint Louis, MO, USA

e-mail: hinckera@wustl.edu 
( $n=8$ months). Because the month-to-month variation was so low, our $95 \%$ confidence intervals (CI) remained of similar size despite the decrease in the number of samples.

This new method of analysis yielded negligibly different effect sizes owing to months with more cases no longer being weighted more heavily in the analysis (e.g., a $20.5 \%$ improvement in the proportion of second doses of antibiotics correctly administered using the new method of analysis $v s$ a $21.1 \%$ improvement using the older method). Confidence intervals for our primary outcome the proportion of second doses of antibiotics administered correctly - were virtually unchanged $(95 \% \mathrm{CI}, 17.3$ to $24.9 \%$ in the original analysis vs 16.2 to $24.7 \%$ in the more recent analysis), although the intervals widened somewhat for the secondary outcomes of the proportions of first and third doses of antibiotics administered correctly.

As Dr. Dexter predicted, the new method of analysis more appropriately characterized the effects of the interventions, but it did not affect the conclusions of the study.
Conflicts of interest None to declare.

Editorial responsibility This submission was handled by Dr. Hilary P. Grocott, Editor-in-Chief, Canadian Journal of Anesthesia.

Financial support and sponsorship This work was supported by the Department of Anesthesiology, Washington University in Saint Louis, Saint Louis, MO, USA.

\section{References}

1. Hincker A, Ben Abdallah A, Avidan M, Candelario P, Helsten D. Electronic medical record interventions and recurrent perioperative antibiotic administration: a before-and-after study. Can J Anesth 2017; 64: 716-23.

2. Dexter, F. Analysis of perioperative antibiotic administration in electronic medical records: correlations among patients addressed by analyzing control chart data using the batch means method. Can J Anesth 2018; 65: DOI:10.1007/s12630-017-0968-z. 